\title{
Téoros
}

Revue de recherche en tourisme

\section{Innovations en tourisme durable}

\section{Rachel Dodds}

Numéro hors-série, 2012

Innovations en tourisme durable

Innovations in Sustainable Tourism

URI : https://id.erudit.org/iderudit/1036555ar

DOI : https://doi.org/10.7202/1036555ar

Aller au sommaire du numéro

Éditeur(s)

Université du Québec à Montréal

ISSN

0712-8657 (imprimé)

1923-2705 (numérique)

Découvrir la revue

Citer ce document

Dodds, R. (2012). Innovations en tourisme durable. Téoros, 3-6.

https://doi.org/10.7202/1036555ar

Ce document est protégé par la loi sur le droit d'auteur. L’utilisation des services d'Érudit (y compris la reproduction) est assujettie à sa politique d'utilisation que vous pouvez consulter en ligne.

https://apropos.erudit.org/fr/usagers/politique-dutilisation/
Cet article est diffusé et préservé par Érudit.

Érudit est un consortium interuniversitaire sans but lucratif composé de l’Université de Montréal, l'Université Laval et l'Université du Québec à Montréal. Il a pour mission la promotion et la valorisation de la recherche. https://www.erudit.org/fr/ 


\title{
Innovations en tourisme durable
}

\author{
Rachel DODDS, Ph.D. \\ Professeure associée \\ Ted Rogers School of Hospitality and Tourism Management \\ Ryerson University \\ r2dodds@ryerson.ca
}

Plusieurs régions dans le monde sont tributaires du tourisme comme moyen de subsistance. Pour nombre d'entre elles, la contribution économique du tourisme ne cesse d'augmenter d'année en année. Bien que le tourisme apporte plusieurs avantages (revenu, emplois, développement économique), de nombreuses destinations connaissent des problèmes importants au niveau environnemental et social, faisant alors du tourisme durable une perspective de développement souhaitable et nécessaire. Même si la littérature est abondante à ce sujet, la mise en œuvre des principes de tourisme durable reste encore timide dans l'industrie touristique. Dans ce contexte, cette édition spéciale de Téoros a pour but de présenter des innovations en matière de tourisme durable, qu'il s'agisse de pratiques réussies ou d'exemples de transfert de connaissances. Les études de cas choisies décrivent différentes innovations en matière de tourisme durable, susceptibles d'inspirer d'autres destinations.

\section{Étudier l'innovation}

À la base, une innovation est largement reconnue comme étant le résultat d'une nouvelle idée créative dont l'application apporte une valeur ajoutée (Sears et Baba, 2011; Yu et Han, 2010). Selon Carlsen et al. (2008: 3), l'innovation peut ainsi être décrite comme " a catalytic development process that activates a number of processes according to a new situation». Elle peut prendre la forme d'un produit, d'un processus, d'une forme de gestion, de logistique ou d'innovation institutionnelle (Hjalager, 1996) et peut être perturbatrice ou progressive (Schaper et Volery, 2007).

La recherche dans le domaine de l'innovation suscite beaucoup d'intérêts en raison de son importante contribution dans tous les secteurs, notamment en lien avec la technologie. Il n'est donc pas étonnant qu'un consensus soit difficile à établir quant à sa définition et son interprétation, en particulier dans le milieu universitaire (Yu et Han, 2010; Markides, 2006; Sears et Baba, 2011; McElroy, 2006). De plus, dans l'industrie du tourisme où les enjeux peuvent être de diverse nature, il est souvent difficile de distinguer les nouvelles idées et concepts pouvant réellement constituer une innovation. Dès lors, ce qui constitue une innovation pour certains peut être vue par d'autres comme étant une adaptation de ce qui existe déjà ou une reproduction d'un concept ayant déjà été développé. Cette question a conduit Hjalager (2002) à suggérer que seules les grandes corporations touristiques seraient capables d'innover car elles sont les seules entités suffisamment puissantes et en mesure d'influencer le fonctionnement de l'industrie dans son ensemble. Dans cette optique, les innovations en matière de tourisme et de développement en niches des entreprises plus modestes seraient alors exclues en raison de leur taille. Pourtant, ces entités sont en mesure d'initier des changements non négligeables, voire révolutionnaires, reflétés par leur nature, leur développement ou leur capacité à partager les connaissances grâce aux partenariats et à l'éducation (Paskaleva-Shapira 2008; Andereck, 2009; McElroy, 2006).

Comme le développement d'innovations présente à la fois des coûts et des bénéfices, il s'avère important de définir ces deux aspects. Les bénéfices identifiés peuvent être classifiés de deux façons. Premièrement, par leur importance stratégique en fonction de quatre catégories de bénéfices : (1) la mise en œuvre; (2) la dynamique de partenariat sociaux; (3) l'efficacité opérationnelle, et (4) la performance économique (O'Connor et al., 1990; Bunduchi et al., 2011). Deuxièmement, les bénéfices peuvent être classés selon qu'ils procurent des avantages directs et indirects ou des avantages relationnels (Weber et Kantamneni, 2002; Bunduchi et al., 2011).

En tourisme, l'innovation résulte souvent de facteurs externes tels que l'évolution démographique, la politique gouvernementale, les changements environnementaux ou les questions sociales. Elle peut aussi être artistique ou conceptuelle dans une perspective visant l'amélioration de la qualité du service ou de l'expérience des visiteurs. Dans ce dernier cas, on parlera d'innovations de valeur (Nankervis et al., 2005). 


\section{Numéro spécial consacré au développement d'innovations}

Cette édition spéciale de Téoros présente différentes innovations à travers le monde - des petites îles touristiques aux grandes régions dans les pays développés et les pays en voie de développement. Cette édition spéciale comprend sept études de cas qui offrent un aperçu des éléments d'importance stratégique associés à différentes catégories de bénéfices. Chaque étude de cas choisie illustre en détail un ou deux éléments particuliers nécessaires à la durabilité : la dynamique de partenariat, le renforcement des capacités, l'éducation et le partage des connaissances, l'accessibilité et les bénéfices pour d'autres intervenants, l'évaluation et la gestion (Bramwell et Lane, 1993; Font et Ahjem, 1999; Liu, 2003; Berresford, 2004; Fadeeva, 2004; Kernel, 2005; Kelman, 2007; Dodds et McElroy, 2008; Williams et Ponsford, 2009; Lim et Cooper, 2009; Graci et Dodds, 2010). De plus, les études de cas englobent plusieurs éléments stratégiques en lien avec la mise en oeuvre, les implications économiques, l'opérationnalisation et la dynamique de partenariat permettant le partage plus large des connaissances entre les destinations. Toutes les études de cas dans ce numéro présentent également les principaux avantages directs et indirects et les relations qui ont été favorisées en raison de ces initiatives. Le but de cette édition spéciale sous forme d'études de cas est donc de souligner l'innovation durable entreprise par les destinations choisies et d'expliquer comment les pratiques adoptées ont des retombées positives pour l'ensemble des parties prenantes.

Chaque étude de cas commence par une mise en contexte sur un enjeu en particulier en matière de tourisme durable suivi d'un cadre de recherche ou d'un bref portrait de la destination puis d'une courte méthodologie. La section subséquente met ensuite en évidence les décisions et les processus pertinents aux dilemmes et enjeux soulevés sous forme de discussion. Elle est suivie d'une section présentant les résultats spécifiques qui illustrent comment la destination ou les acteurs étudiés ont dénoué les problèmes ou ont surmonté les difficultés et quels aspects de leur intervention étaient innovateurs. Compte tenu de l'éventail des contributions à cette édition spéciale, une série de thèmes associés aux conditions principales du tourisme durable a été identifiée, comme mentionnée précédemment. Chaque contributeur se concentre sur un aspect particulier pour éviter la répétition et discute principalement de l'approche et des résultats. En effet, Hjalager et al. (2008: 33) estiment que l'innovation nécessite la combinaison et le mariage d'idées qui se développent le mieux par le réseautage et une panoplies d'autres liens. Ainsi, cette publication vise à diffuser l'innovation dans les organisations et les destinations en offrant un cadre d'analyse et de discussion.

Les contributeurs ont participé à un atelier conjoint français/anglais qui s'est tenu à Toronto en mars 2012. Étudiantes et étudiantes de maîtrise et professeurs travaillant dans le domaine du tourisme se sont réunis pour partager leurs connaissances. Ils ont été invités à produire une étude de cas pertinente pour un public international et qui démontre la pertinence de l'innovation et des processus ou cadres de gestion utilisées pour atteindre les objectifs visés. Les études de cas se veulent courtes (environ 3000 mots). Néanmoins, les auteur(e)s ont été invité(e)s à fournir une brève revue de la littérature sur le sujet et une analyse historique ou descriptive des processus et des avantages acquis transférables vers d'autres destinations. Finalement, ce numéro de Téoros a été produit à la fois en français et en anglais pour combler le manque de littérature bilingue dans le domaine du tourisme et pour partager la recherche appliquée dans l'espoir d'intégrer l'innovation dans la recherche et les pratiques territoriales.

\section{Les études de cas}

Les sept études de cas de ce numéro présentent chacune différents avantages. Premièrement, on y analyse les retombées directes et indirectes à travers deux études de cas qui mettent l'accent sur les petites collectivités. Dans le premier article, Kelly MacKay et J. Michael Campbell relatent le renforcement des capacités en tourisme communautaire en Ouganda et l'importante des partenariats locaux et nationaux pour venir en aide aux populations locales en contribuant à la durabilité de leurs opérations et activités. Les camps de tentes en Ouganda montrent que même à petite échelle, la performance économique est susceptible de bénéficier à l'ensemble de la communauté à travers un modèle de partage des profits et de développement de l'éducation. L'innovation, dans ce premier article, repose sur une approche triangulée de l'éducation, de la participation communautaire et des réseaux stratégiques.

Dans le second article, Sonya Graci présente le cas de l'écolodge canadien Moose Cree et démontre les avantages directs et indirects résultant du développement du tourisme. Elle y observe l'amélioration de la dynamique communautaire et les avantages économiques résultants. Le développement du tourisme communautaire de cet écolodge de renommée mondiale a ainsi permis l'autonomisation des populations locales, gage de moyens de subsistance durables. Comme dans le cas du Cree Village Ecolodge, la communauté a bénéficié d'un développement économique majeur en misant sur sa fierté culturelle. Ce développement économique est marqué par une dépendance réduite à l'aide gouvernementale et une préservation du patrimoine naturel et culturel

Ensuite, deux articles s'attardent principalement sur les innovations en matière de mesure et d'évaluation en tourisme durable, respectivement dans une région insulaire en développement et une péninsule d'un pays développé. Ainsi, dans le troisième article, Lacey Willmott et Sonya Graci discutent du cas de l'île indonésienne de Gili Trawangan. Les auteures démontrent que la mise en place d'un partenariat multi-parties prenantes bénéficie aux entreprises de la région et à l'ensemble de la destination. Elle peut ainsi conduire au développement d'un système de gestion peu commun dans les pays en développement. Leur article se concentre la gestion des matières résiduelles attribuables au tourisme Ces dernières sont à l'origine de plusieurs problèmes environnementaux et de la pollution esthétique néfaste à l'expérience touristique et à la communauté hôte. Un réseau de partenariats stratégiques illustre la façon dont un partenariat peut augmenter l'efficacité de la gestion des matières résiduelles grâce à l'établissement des principes de la séparation à la source et du stockage, essentiels à l'efficacité du système de collecte. Cent pour cent des résidents de l'île reçoivent désormais un service 
de collecte des déchets avec une amélioration du processus de planification de la collecte. L'innovation réside dans l'engagement des autorités responsables à faciliter le dialogue entre les Indonésiens et les Occidentaux et dans la création d'un partenariat nécessaire pour un système de gestion des matières résiduelles efficace.

Dans le quatrième article, Juste Rajaonson et Georges Tanguay illustrent comment un système d'évaluation du tourisme durable basé sur des indicateurs développés à une échelle globale peut se révéler efficace au niveau local. Leur article décrit l'approche utilisée par les régions de la Gaspésie et des Îles de la Madeleine, au Québec, pour établir une grille d'indicateurs de tourisme durable en mesure d'appuyer leur stratégie politique. Cette stratégie innovante permet d'identifier une liste parcimonieuse d'indicateurs de tourisme durable à la fois pertinents, mesurables et cohérents avec la politique touristique de chaque région. Le résultat met en valeur la façon dont l'expertise scientifique internationale a été utilisée à un niveau local pour améliorer l'efficacité de gestion et la transparence.

Dans le cinquième article, Bruno Sarrasin et Jonathan Tardif apportent une vision plus large de la gestion du tourisme et cherche à savoir si la co-gestion pourrait être analysée comme une pratique novatrice dans la promotion des ressources naturelles axée sur le tourisme. Le projet de Sentier national Waitukubuli effectué à la Dominique en est un exemple d'une pratique innovatrice de co-gestion des ressources éco-touristiques. La participation et l'implication locale dans la planification du tourisme par les multiples parties prenantes ont conduit au développement de corridors touristiques entre les segments du sentier et les communautés marginalisées. Ainsi, ces corridors ont tiré profits du tourisme bien que leur vocation visait essentiellement la protection écologique.

Dans les deux derniers articles, Rachel Dodds et Nicolas Berthet examinent la mise en ouvre spécifique du tourisme durable ayant permis des retombées socioéconomiques et environnementales plus larges via le transfert des connaissances. Des facteurs externes tels que les changements politiques, démographiques ou environnementaux peuvent avoir des incidences importantes sur le développement d'une région. Heureusement, les innovations au niveau régional ou national permettent de surmonter ces circonstances négatives éventuelles. D'une part, Nicolas Berthet discute d'une démarche politique ayant eu des retombées positives importantes dans le département français des Pyrénées-Orientales (aussi appelé Catalogne nord ou française) et servant de référence à d'autres départements et régions. Le Conseil Général des PyrénéesOrientales a su démontrer qu'au-delà de ses avantages économiques, une politique de transport durable contribue aussi à réduire les problèmes de saisonnalité à augmenter l'accessibilité à l'arrière-pays. L'innovation principale a été le rôle joué par le Conseil Général et les parties prenantes du tourisme local dans la protection et la mise en valeur du patrimoine historique, culturel et naturel grâce à une accessibilité renforcée par la mise en place d'un réseau de transport en commun plus performant.

Finalement, l'article de Rachel Dodds au sujet de l'île de Chumbe en Tanzanie met en perspective la façon dont la conservation et la gestion efficaces ont profité aux communautés locales ainsi qu'à l'ensemble du pays, grâce à au développement de partenariats. La sensibilisation environnementale offerte aux touristes, aux enseignant(e)s et aux étudiant(e)s tanzanien(ne)s a facilité le partage des connaissances à l'échelle de l'̂̂le. Elle a eu tellement de succès que le cadre éducatif est maintenant utilisé à l'échelle nationale.

\section{Sommaire}

Les sept études de cas ci-après soulignent des exemples provenant de différentes régions du monde ainsi que de destinations de différentes tailles et de différents contextes socioéconomiques. Malgré leur diversité, chaque étude de cas met en valeur les innovations d'importance stratégique en lien avec quatre catégories d'avantages, au niveau (1) de la mise en œuvre; (2) de la dynamique des partenariats sociaux; (3) de l'efficacité opérationnelle et (4) de la performance économique. Les avantages directs, indirects et relationnels sont présentés dans l'intention d'encourager le transfert des connaissances afin de contribuer au développement du tourisme durable dans le monde.

\section{Références}

ANDERECK, Kathleen L. (2009) « Tourists' perceptions of environmentally responsible innovations at tourism businesses ", Journal of Sustainable Tourism, vol. 17, $\mathrm{n}^{\circ} 4$, p. 489-499.

BERRESFORD, James (2004) "Tourism in the region", Regional Review Hearing, p. 1-11.

BRAMWELL, Bill et Bernard Lane (1993) «Sustainable tourism: an evolving global approach», Journal of Sustainable Tourism, vol. 1, nº 1, p. 1-4.

BUNDUCHI, Raluca; Clara WEISSHAAR et Alison U. SMART

(2011) «Mapping the benefits and costs associated with process innovation: the case of RFID adoption ", <http://www.abdn.ac.uk/ cms152/ TECH2011.pdf >, consulté le 01.02.2011.

CARLSEN, Jack; Janne LIBURD: Deborah EDWARDS et Paddy FORDE (2008) «Overview of innovation for sustainable tourism», Dans CARLSEN, Jack; Janne LIBURD; Deborah EDWARDS et Paddy FORDE (sous la direction de), Innovations for Sustainable Tourism: International Case Studies, p. 3-15, BEST, Australie.

DODDS, Rachel et Jerome L. MCELROY (2008) «St. Kitts at a crossroads». ARA Journal of Travel Research, vol. 1, n 2, p. 1-10.

FADEEVA, Zinaida (2004) «Translation of sustainability ideas in tourism networks: some roles of cross-sectoral networks in change towards sustainable development» Journal of Cleaner Production, vol. 13, $\mathrm{n}^{\circ} 2$, p. 175-189.

FONT, Xavier et Tor E. AHJEM (1999) «Searching for a balance in tourism development strategies ", International Journal of Contemporary Hospitality Management, vol. 11, n 2/3, p. 73-77.

GRACI, Sonya et Rachel DODDS (2010) Sustainable tourism in island destinations. Earthscan, Londres, 248 p.

HJALAGER, Anne-Mette (1996) «Tourism and the environment: the innovation connection ", Journal of Sustainable Tourism, vol. 4, $\mathrm{n}^{\circ} 4$, p. 201-218

HJALAGER, Anne-Mette (2002) «Repairing innovation defectiveness in tourism », Tourism Management, vol. 23, n 5, p. 465-474.

HJALAGER, Anne-Mette; E.H. HUIJBENS; P. BJÖRK; S. NORDIN; A. FLAGESTAD et Ö. KNÚTSSON (2008) Innovation systems in Nordic tourism. Nordic Innovation Centre, Oslo. 72 pages. 
KELMAN, Ilan (2007) «Sustainable livelihoods from natural heritage on islands », Island Studies Journal, vol. 2, n 1, p. 101-114.

KERNEL, Pernille (2005) «Creating and implementing a model for sustainable development in tourism enterprises ", Journal of Cleaner Production, vol. 13, n 2, p. 151-164.

LIM, Charles C. et Chris COOPER (2009) «Beyond sustainability: optimising island tourism development», International Journal of Tourism Research, vol. 11, n 1, p. 89-103.

LIU, Zhenhua (2003) «Sustainable tourism development: a critique», Journal of Sustainable Tourism, vol. 11, $\mathrm{n}^{\circ}$ 6, p. 459-475.

MARKIDES, Constantinos (2006) «Disruptive innovation: in need of better theory", Journal of Product Innovation Management, vol. 23, $\mathrm{n}^{\circ} 1$, p. $19-25$.

MCELROY, Jerome L. (2006) «Small Island Tourism Economies Across the Life Cycle», Asia Pacific Viewpoint, vol. 47, n 1, p. 61-77.

NANKERVIS, Alan; Yuki MIYAMOTO; John MILTON-SMITH et Ruth TAYLOR (sous la direction de) (2005) The Management of Services, Cambridge University Press, Cambridge. 376 p.

O'CONNOR, Edward J.; Charles K. PARSONS; Robert C. LINDEN et David M. HEROLD (1990) «Implementing New Technology: Management Issues and Opportunities », Journal of High Technology Management Research, vol. 1, n 1, p. 69-89.
PASKALEVA-SHAPIRA, Krassimira; Jose AZORIN et Aline CHIABAI (2008) «Enhancing Digital Access to Local Cultural Heritage Through e-Governance: Innovations in Theory and Practice from Genoa, Italy", Innovation: The European Journal of Social Sciences, vol. 21, $\mathrm{n}^{\circ} 4, \mathrm{p}$. 389- 405.

SEARS, Greg J. et Vishwanath V. BABA (2011) «Toward a multistage, multilevel theory of innovation ", Canadian Journal of Administrative Sciences, vol. $28, \mathrm{n}^{\circ} 4$, p. $357-372$.

SCHAPER, Michael et Thierry VOLERY (2007) Entrepreneurship and Small Business. $2^{\text {nd }}$ Pacific Rim Edition. Wiley, Stafford, Australie, 482 p.

YU, Dan et Chang Chieh HANG (2010) «A Reflective Review of Disruptive Innovation Theory", International Journal of Management Reviews, vol. $12, \mathrm{n}^{\circ} 4$, p. $435-452$.

WEBER, Mary Margaret et S. Prasad KANTAMNENI (2002) «POS and EDI in retailing: an examination of underlying benefits and barriers ", Supply Chain Management, vol. 7, $\mathrm{n}^{\circ}$ 5, p. 311-317.

WILLIAMS, Peter W. et Ian F. PONSFORD (2009) «Confronting tourism's environmental paradox: Transitioning for sustainable tourism », Futures, vol. $41, n^{\circ} 6$, p. 396-404.

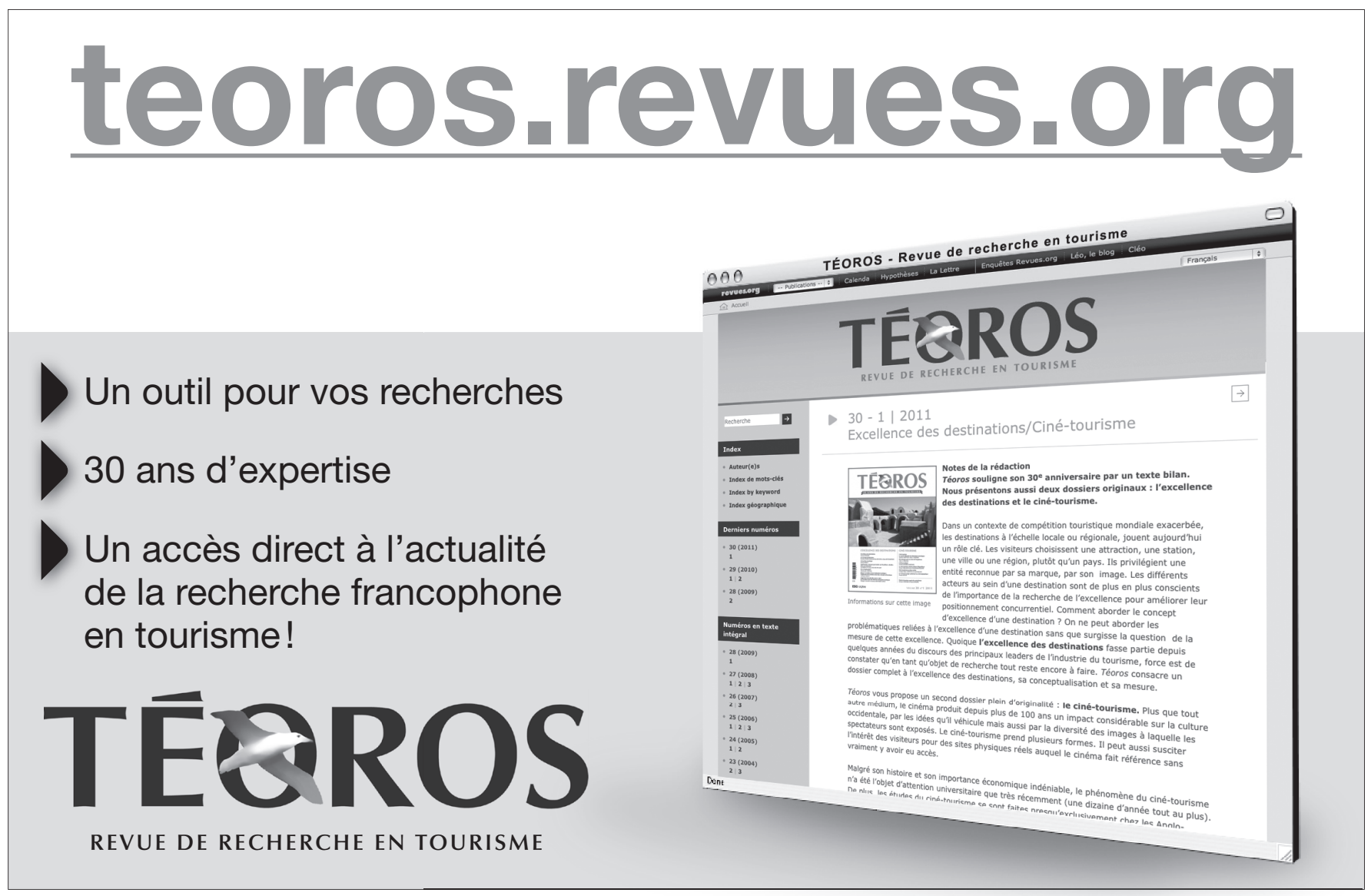

TÉOROS, hors série, p. 3-6 @ 2012 